\title{
昡pubvet
}

https://doi.org/10.31533/pubvet.v14n9a651.1-9

\section{Intoxicação por Cannabis sativa: Desafios relacionados à clínica de animais de companhia}

\author{
Otávio Luiz Cardoso Rios ${ }^{19}$, Kelmanny Crislen Ferreira Sousa ${ }^{19}$, Thiago Barros Correia da

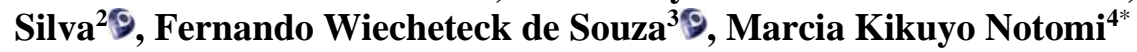

${ }^{I}$ Discente do Curso de Medicina Veterinária da Universidade Federal de Alagoas

${ }^{2}$ Professor associado de Farmacologia da Universidade Federal de Alagoas, Curso de Medicina Veterinária. Viçosa -AL Brasil.

${ }^{3}$ Professor adjunto de Cirurgia da Universidade Federal de Alagoas, Curso de Medicina Veterinária. Viçosa -AL Brasil.

${ }^{4}$ Professora associada de Clínica da Universidade Federal de Alagoas, Curso de Medicina Veterinária. Viçosa-AL Brasil.

*Autor para correspondência: E-mail: marcia.notomi@vicosa.ufal.br

Resumo. As plantas do gênero Cannabis produzem, entre outros, uma classe de compostos químicos com relevante ação psicotrópica, os fitocanabinoides, por este motivo ela é a droga recreativa de maior consumo mundial. A proximidade dos animais de companhia com humanos e seus aspectos comportamentais, os tornam suscetíveis a exposição a essa e outras drogas. O perfil toxicológico da intoxicação por Cannabis sativa L. está diretamente relacionada à proporção entre a concentração do delta-9-tetraidrocanabinol e canabidiol na amostra ingerida. Dois compostos químicos com elevada lipossolubilidade, o que faz sua absorção, distribuição e metabolização ocorrer rapidamente. Seguida a absorção, eles interagem com receptores canabinoides presente no corpo, o CB1 (predominante no cérebro) é o responsável pela maior parte dos sintomas causados pelo contato, ocasionando principalmente sintomas relacionados ao sistema nervoso central. Contudo, a interação com o CB2 (predominante em outras partes do corpo) e o perfil variável dessa interação com ambos receptores, que pode resultar em um efeito excitatório ou inibitório, dificultando a realização do diagnóstico clínico, sendo agravado pela ausência de teste rápido compatíveis para o diagnóstico de intoxicação. O tratamento, diante da ausência de antídotos específicos, se baseia na redução da absorção, terapia de suporte baseado na sintomatologia clínica presente e na peculiaridade das intoxicações por Cannabis. Na ausência de afecções concomitantes o prognóstico é favorável, porém é importante seu reconhecimento e diferenciação de outras intoxicação e afecções neurológicas.

Palavras chave: Canino, droga, fitocanabinoides, maconha, THC

\section{Cannabis sativa poisoning: Challenges related to the pet clinic}

Abstract. Plants of the genus Cannabis produce, among others, a class of chemical
compounds with relevant psychotropic action, phytocannabinoids, for this reason it is the
recreative drug with the greatest consumption worldwide. The proximity of pet animals to
humans and their behavioral aspects make them susceptible to exposure to this and other
drugs. The toxicological profile of Cannabis sativa L. intoxication is directly related to the
proportion between the concentration of delta-9-tetrahydrocannabinol and cannabidiol in
the ingested sample. Two chemical compounds with high liposolubility, which makes their
absorptions, distribution and metabolization occur quickly. Following absorption, they
interact with cannabinoid receptors present in the body, CB1 (predominant in the brain) is
responsible for most of the symptoms caused by contact, causing mainly symptoms related
to the central nervous system. However, the interaction with CB2 (predominant in other
parts of the body) and the variable profile of this interaction with both receptors can result 
in an excitatory or inhibitory effect, making the clinical diagnosis difficult, being aggravated by the absence of compatible rapid tests for the diagnosis intoxication. Treatment, in the absence of specific antidotes, is based on reduced absorption, supportive therapy based on the present clinical symptoms and the peculiarity of Cannabis intoxications. In the absence of concomitant conditions, the prognosis is favourable, but its recognition and differentiation from other intoxication and neurological disorders is important.

Keywords: Canine, drug, phytocannabinoids, marijuana, THC

\section{Intoxicación por Cannabis sativa: desafíos relacionados con la clínica de animales de compañía}

Resumen. Las plantas del género Cannabis producen, entre otros, una clase de compuestos químicos con acción psicotrópica relevante, fitocannabinoides, por esta razón es la droga de recreación con mayor consumo en todo el mundo. La proximidad de los animales de compañía con los humanos y sus aspectos de comportamiento los hacen susceptibles a la exposición a esta y otras drogas. El perfil toxicológico de la intoxicación por Cannabis Sativa L. está directamente relacionado con la proporción entre la concentración de delta9-tetrahidrocannabinol y cannabidiol en la muestra ingerida. Dos compuestos químicos con alta liposolubilidad, que hacen que sus absorciones, distribución y metabolización ocurran rápidamente. Después de la absorción, interactúan con los receptores de cannabinoides presentes en el cuerpo, CB1 (predominante en el cerebro) es responsable por mayoría de los síntomas causados por el contacto, ocasionando principalmente síntomas relacionados al sistema nervioso central. Sin embargo, la interacción con CB2 (predominante en otras partes del cuerpo) y el perfil variable de esta interacción con ambos receptores puede dar lugar a un efecto excitador o inhibidor, lo que dificulta el diagnóstico clínico y se agrava por la ausencia de pruebas rápidas compatibles para el diagnóstico. Intoxicación. El tratamiento, en ausencia de antídotos específicos, se basa en una absorción reducida, una terapia de apoyo basada en los síntomas clínicos presentes y la peculiaridad de las intoxicaciones por Cannabis. En ausencia de condiciones concomitantes, el pronóstico es favorable, pero su reconocimiento y diferenciación de otras intoxicaciones y trastornos neurológicos es importante.

Palabras clave: Canino, drogas, fitocannabinoides, marihuana, THC

\section{Introdução}

Os animais de companhia, principalmente os cães, podem ser expostos a diversas drogas de abuso, como anfetaminas, opioides, cocaína, drogas psicoativas e principalmente a Cannabis (cânabis ou maconha). As circunstâncias que envolvem a exposição, podem variar desde um tutor pouco cuidadoso que facilita o acesso, administração intencional da droga ou cães policiais em treinamento que inadvertidamente ingerem/ inalam o produto (Bates, 2018).

As plantas do gênero Cannabis produzem uma classe única de compostos químicos, os canabinoides, que atuam em receptores específicos (CB1 ou CB2), produzindo um efeito psicotrópico (Borille, 2016; Landa et al., 2016). São encontrados em maior concentração nos tricomas glandulares destas plantas, sendo que o delta-9-tetrahidrocanabinol (THC) é o mais numeroso (Aguiar, 2018; Farag \& Kayser, 2017) . No cérebro, a interação entre THC e CB1 causa uma alteração no padrão de funcionamento de diversos neurotransmissores, podendo desencadear um efeito estimulatório ou inibitório (Botha \& Penrith, 2009).

A intoxicação por Cannabis pode ocorrer por via digestória ou inalatória, apresentando sintomatologia variável conforme a via por onde ocorreu a intoxicação, quantidade de THC presente na amostra com a qual ocorreu o contato, entre outros fatores (Parshley \& Mensching, 2014). Apesar da inexistência de antídoto específico, essa intoxicação possui prognóstico favorável na ausência de 
complicações secundárias. Seu tratamento buscar fornecer suporte ao paciente, adequando-se conforme o quadro de sintomas apresentado, reduzindo os riscos e os danos causados (Miranda et al., 2017).

O principal desafio para o profissional constitui em obter um diagnóstico conclusivo, visto que para realizá-lo é necessária a identificação do metabólito 11-OH-delta-9-tetrahidrocanabinol (11-OH-THC) na urina (produto da conversão do THC nos pulmões e fígado). Como agravante, os cães possuem uma via adicional de beta-oxidação que converte o THC em 8-OH-delta-9-tetrahidrocanabinol (8-OH-THC), ocasionando falso-negativos na espécie canina, com o uso do teste rápido disponível para humanos (Miranda et al., 2017). Em conjunto a esse aspecto, deve-se levar sempre em consideração que, por se tratar de uma substância ilícita, recomenda-se cautela nos termos utilizados durante a anamnese, de modo a não desestimular o tutor a fornecer os dados necessários para o diagnóstico correto (Janczyk et al., 2004; Luiz \& Heseltine, 2008).

Considerando o contexto citado, o presente trabalho tem como objetivo realizar uma revisão de literatura, atualizando sobre intoxicações por Cannabis na clínica de animais de companhia, visando auxiliar na prevenção das intoxicações, no rápido diagnóstico e na escolha do tratamento adequado.

\section{Desenvolvimento}

A Cannabis é a droga mais consumida mundialmente (Borille, 2016), sendo estimado em 2013, um número de usuários de 3,9\% da população mundial, cerca de 180,6 milhões de pessoas com idade entre 15 a 64 anos (Nascimento, 2014). Llera \& Volmer (2006) relatam que a Cannabis é a droga que os cães policiais correm mais riscos de exposição, devido sua popularidade como droga recreativa, o que a torna facilmente acessível.

A principal via de intoxicação é através da ingestão de folhas e flores da planta e seus derivados incorretamente armazenadas pelos seus tutores (Ferreira et al., 2013). Porém, outra via possível de intoxicação é a respiratória, através da inalação da fumaça produzida pela queima da planta (Meola et al., 2012).

No Brasil, Hansen (2006) relatou que dos 120 casos de cães intoxicados, no período de novembro de 2004 a outubro de 2005, quatro destes ocorreram pelo contato com Cannabis. A Animal Poison Control Center (EUA), tem o registro de mais de 250 casos de intoxicação por Cannabis entre 1998 a 2002, sendo a maior incidência em cães ( $96 \%$ dos casos), seguido por gatos (3\% dos casos) e outras espécies acumulam 1\% das ocorrências (Donaldson, 2002). Botha \& Penrith (2009), na África do Sul, verificaram 213 cães intoxicados, sendo 10 decorrentes da ingestão de biscoitos contendo THC e 203 de folhas ou cigarros de maconha. ASPCA Animal Poison Control Centre (EUA) registrou 213 intoxicações por via digestória, no período de 1998 a 2002, sendo quatro por comerem brownies contendo THC, seis por comerem biscoitos com THC e 203 por ingestão de folhas ou cigarros de maconha (Janczyk et al., 2004).

A quantidade ingerida nos casos de intoxicações pode variar em razão da concentração da droga. Um estudo realizado no estado de São Paulo, analisou 28 amostras apreendidas pela polícia local e encontrou uma concentração que variou entre 0,5 a $1,55 \%$ de THC, sendo que a concentração da droga pode chegar a até $60 \%$ de THC (Oliveira, 2005).

Os canabinóides são compostos altamente lipossolúveis, por exemplo, o THC possui um coeficiente de partição octanol-água de 600:1. Esta propriedade relaciona-se diretamente aos efeitos biológicos causados pelo contato com estas classes de compostos químicos, devido sua rápida absorção e capacidade de se acumular na membrana celular (Piedra et al., 2009). É importante destacar que os efeitos fisiológicos do THC no cérebro duram mais tempo que sua concentração sérica (Ribeiro, 2014).

Quando a ingestão ocorre por via oral, a biodisponibilidade pode variar de acordo com a perda ocorrida pela ação do suco gástrico e pela presença de lipídeos, que aumenta a absorção em até 95\%. Após entrar na circulação sanguínea, o THC se une principalmente a lipoproteínas de baixa densidade, uma menor parte se liga a albumina ou células sanguíneas (Piedra et al., 2009). Em seguida é distribuído na gordura, no fígado, no cérebro e no rim. $\mathrm{O}$ armazenamento em gordura faz com que o THC tenha uma meia vida de 30 horas (Fitzgerald et al., 2013).

O delta-9-THC é o canabinóides com maior potência psicoativa, este canabinóides é um composto não cristalino de elevada lipofilia, o que lhe facilita a adsorção no organismo e consequentemente uma 
maior rapidez de ação (Ribeiro, 2014). A administração por via pulmonar, mais eficiente em velocidade de absorção e biodisponibilidade que a via oral, leva ao pico de concentração de CBD e THC no soro e no cérebro nos primeiros minutos após contanto, quando por via oral, o pico de concentração só é alcançado após duas horas (Aguiar, 2018).

O THC tem um padrão farmacocinético multicomportalmental, com uma fase de distribuição e uma súbita caída nas concentrações plasmáticas. A distribuição inicia imediatamente após a absorção. No sangue, ele se liga as proteínas plasmáticas, fazendo que chegue primeiro nos tecidos mais irrigados, como rim, pulmões, fígado, cérebro, entre outros; em seguida ele se acumula no tecido adiposo. A natureza lipofílica também permite ao THC atravessa a barreira placentária, onde o feto terá uma concentração plasmática equivalente a $10 \%$ da presente na mãe, causando danos à sua saúde (Huestis, 2005; McGilveray, 2005; Piedra et al., 2009).

No cérebro o THC interage com neurotransmissores e neuromodulares (Luiz \& Heseltine, 2008) A interação entre o ele e o CB1 altera o padrão de funcionamento de diversos neurotransmissores (dopamina, serotonina, noradrenalina, ácido gama-aminobutirico, entre outros), podendo causar um efeito estimulatório ou inibitório (Botha \& Penrith, 2009; Lee, 2014).

Quando um canabinóide interage com um receptor canabinóide, as proteínas G são ativadas, logo abrem-se ou fecham-se os canais de cálcio e potássio, alterando a funções do SNC. O THC e a anandamida, seu agonista endógeno, inibem a enzima adenilato ciclase, diminuindo a produção de adenosina monofosfato cíclica, o que causa a abertura dos canais de potássio e a oclusão dos canais de cálcio, reduzindo a liberação de neurotransmissores (Ribeiro, 2014).

O metabolismo do THC ocorre em diversos tecidos, como cérebro, intestino e pulmão, mas a principal via ocorre por meio da hidroxilação do THC pela enzima hepática citocromo P450, que resulta em mais de 100 metabólitos (cetonas, aldeídos, entre outros), dentre os quais, aponta-se o 11-OH-THC como predominante, podendo ser encontrado em níveis plasmáticos que se aproximam em $10 \%$ dos níveis de THC (Huestis, 2005). O THC é rapidamente metabolizado pelo fígado, convertido em 11-OHTHC, que é oxidado em 11-COOH-THC (Janeczek et al., 2018).

Em humanos, cerca de 90\% do THC absorvido é eliminado após 5 dias, maior parte como metabólitos hidroxilados e carboxilados, cerca de $65 \%$ com as fezes e cerca de $20 \%$ com a urina. Diversos metabólitos ácidos foram encontrados na urina, muitos conjugados ao ácido glucurônico para ficarem mais hidrossolúveis ( urinária (Fitzgerald et al., 2013).

Os sinais clínicos são poucos claros, vistos que os receptores canabinoides possuem um comportamento bifásico (variando conforme a dose). Nos cães, as drogas causam sintomas similares aos apresentados por humanos, porém mais difíceis de interpretar. Para agravar esta situação, não existe teste rápido valido para a espécie, restando aos veterinários o seu julgamento clínico para o diagnóstico e implantação de tratamento (Grotenhermen, 2004; Turner et al., 1980).

A ingestão de plantas com níveis elevados de THC e reduzido de CBD irá produzir uma ação psicoativa forte e clara, plantas com ambos níveis elevados produzem uma intensa modificação do sistema nervoso central e as que possuírem um baixo teor de THC e alto de CBD causarão uma ação que tende a tranquilização e relaxamento do corpo (Nascimento, 2014).

Os sintomas tendem a ser mais amenos quando o contato se dá por via inalatória, quando comparados com as manifestações por intoxicações via digestória (Lee, 2014). Podem ser predominantemente neurológicos, gastrointestinais ou ambos (Botha \& Penrith, 2009), em menor proporção, podem se relacionar ao sistema cardiorrespiratório ou como uma miscelânea de sintomas relacionados a outros sistemas (Turner et al., 1980).

Além de alterações nos padrões clínicos por fatores correlacionados a droga, a via por onde ocorreu a exposição e fatores intrínsecos ao paciente também irão interferir na sintomatologia, por exemplo, pacientes do sexo feminino costumam apresentar maior sensibilidade ao THC, decorrente da sua interação com o estrogênio (Parshley \& Mensching, 2014; Ribeiro, 2014). 
Apesar da alta dose letal, maior que $3 \mathrm{~g} / \mathrm{kg}$, o contanto com a droga numa dose de $0,5 \mathrm{mg} / \mathrm{kg}$ é motivo bastante para que cães e gatos manifestem sinais clínicos (Mckinney, 2017). A sintomatologia costuma aparecer entre 30 a 90 minutos seguidos do contato (Luiz \& Heseltine, 2008) e podem permanecer por até 72 horas, já que o THC é altamente lipofílico e pode sofre recirculação enterohepática (Wismer, 2016).

A severidade do quadro clínico está diretamente relaciona à quantidade de THC que o paciente foi exposto (Luiz \& Heseltine, 2008). Tal fator relaciona-se ainda com a via por onde ocorreu a intoxicação, além de fatores fisiológicos como a velocidade de metabolização e excreção. Animais intoxicados por via respiratória tendem recuperar-se em poucas horas, enquanto os que tiveram contato com uma pequena quantidade por via digestória, tendem a necessitar de 24 horas para recuperação, e os que ingeriram uma grande quantidade, podem manifestar sintomatologia por alguns dias (Bischoff, 2018).

Botha \& Penrith (2009) relataram 213 casos, onde 99\% que manifestaram sintomas relacionado ao sistema neurológico e $30 \%$ ao gastrointestinal, o que prevalece também no relato de Janczyk et al. (2004), onde em 230 casos de cães intoxicados, 99,1\% apresentaram sinais neurológicos e 30,5\% gastrointestinais. Os principais sinais observados em ambos estudos foram depressão (60-61\%), ataxia ou incoordenação (59\%); êmese (24-25\%); tremores (18-25\%) e midríase (10 a 11\%).

Meola et al. (2012) analisou 125 casos, no Colorado, EUA e os sinais clínicos mais observados foram: ataxia (88\%), desorientação $(53 \%)$, midríase $(48 \%)$, incontinência urinaria $(47 \%)$, hiperestesia (47\%), tremores (30\%) e êmese (27\%). Este relato apresentou dois casos de óbito, ambos com pacientes que ingeriram doses significativamente menor que a dose letal, o autor levantou a hipótese dos pacientes serem mais sensíveis ao THC, apesar de afirmar que a ingestão de THC não foi motivo suficiente para os óbitos nos dois casos, a ingestão pode ter contribuído significativamente.

Wismer (2016) cita letargia, depressão, ataxia e incontinência urinária como os sinais clínicos mais comuns, seguidos por hiperexcitabilidade, hiperestesia, tremores, bradicardia, hipotermia e midríase. Parshley \& Mensching (2014) destacam ainda o risco de complicações secundárias, como trauma decorrente da incoordenação ou pneumonia aspirativa, pela combinação entre êmese e depressão do SNC.

Os demais sintomas apareceram com uma ocorrência inferior a 10\% dos casos; sendo descrito sialorreia, incontinência urinária, fraqueza, head pressing, desorientação, hiperexcitabilidade, coma, pulso irregular e hipertermia (Botha \& Penrith, 2009). Além de hipotermia, fraqueza, bradicardia, desorientação, sialorreia, alterações comportamentais, hiperestesia, vocalização, anorexia, incontinência urinária, taquicardia, hipertermia, coma, hiperatividade, tremores na cabeça, entre outros (Janczyk et al., 2004).

O diagnóstico de intoxicação por drogas em animais de companhia é um desafio ao médico veterinário, o caráter ilegal de tais substâncias pede ao profissional um maior cuidado ao realizar a anamnese. $\mathrm{O}$ tutor pode desconhecer a exposição em casos onde adolescentes são os responsáveis pela intoxicação ou, tendo ciência da situação, negar-se a fornecer informações (Bischoff, 2018; Luiz \& Heseltine, 2008).

A anamnese se torna uma ferramenta de diagnóstico ainda mais importante em cães, já que o kit para diagnóstico utilizado para triagem de THC, baseia-se na presença do metabólito 11-OH-THC e 11COOH-THC, ambos derivados da degradação hepática e pulmonar do THC. Este teste demonstrou-se eficaz para o diagnóstico em gatos, contudo, cães possuem uma via alternativa de beta-oxidação para degradar o THC em 8-OH-THC, que pode acarretar em um falso negativo para testes nesta espécie, já que o teste não identifica este metabólito, sendo, portanto, desaconselhado a utilização do teste ELISA em pacientes caninos (Fernández \& Pretti, 2017; Janeczek et al., 2018; Meola et al., 2012; Parshley \& Mensching, 2014).

Fernández \& Pretti (2017) indicam a coleta de conteúdo gástrico, para análise laboratorial afim de encontrar resquícios de intoxicação por Cannabis pela via digestória. Wismer (2016) considera essencial realizar testes para etilenoglicol, de forma a eliminar a possibilidade de tal intoxicação, que nos estágios iniciais, sendo similar a intoxicação por maconha. Teitler (2009) ressalta a importância de um 
diagnóstico rápido para quadros emergenciais, afirmando que outros métodos de diagnósticos mais demorados servem apenas para propósitos legais e médicos.

Por padrão, o diagnóstico de intoxicação por Cannabis é clínico, baseando-se nos sinais clínicos presentes e na realização de uma boa anamnese e exame físico. Para isso, é necessário que o veterinário tenha um bom preparo para identificar os sinais e ser cuidadoso ao questionar o tutor (Hansen, 2006; Parshley \& Mensching, 2014). O veterinário deve considerar ainda um conjunto de possíveis diagnósticos diferenciais, como etanol, etilenoglicol, barbitúricos, ivermectina, opioides, benziazepínicos, fenotiazínicos, relaxantes musculares, etc (Donaldson, 2002; Fitzgerald et al., 2013).

Ainda que confirmada a intoxicação por Cannabis, o profissional veterinário deve considerar a possibilidade de outros fatores estarem prejudicando o prognóstico do paciente, tentar questionar ao tutor se outras drogas de abusos ou demais substâncias intoxicantes estão envolvidas (Mahaney, 2011).

Diante da inexistência de antídotos específicos que revertam os efeitos da intoxicação por plantas do gênero Cannabis, o tratamento para tais casos se baseia em reduzir a absorção do agente, tratar os sintomas apresentados e oferecer uma terapia de suporte ao paciente (Fernández \& Pretti, 2017; Ferreira et al., 2013; Fitzgerald et al., 2013).

A indução de êmese é recomendada na primeira hora após contato com a droga e apenas nos casos onde o paciente não apresentam sinais que possam facilitar uma pneumonia aspirativa (relacionados a depressão no SNC). Em gatos, recomenda indução de êmese utilizando a xilazina $(1,1 \mathrm{mg} / \mathrm{kg}$ por via intramuscular), em cães recomenda-se apomorfina $(0,04 \mathrm{mg} / \mathrm{kg}$ por via intravenosa ou intramuscular). Apesar das duas serem a droga de primeira escolha para indução de êmese em suas respectivas espécies, ambas podem falhar devido às alterações causadas pelo THC na região do SNC que controla o reflexo de vômito, nesses casos recomenda-se a utilização de um irritante local, como o peróxido de hidrogênio, que deve ser usado na concentração igual ou inferior a 3\% (para evitar lesão da mucosa gástrica), numa dose entre 2 a $5 \mathrm{mg} / \mathrm{kg}$, não excedendo a dose total de $50 \mathrm{mg}$ nos cães e $10 \mathrm{mg}$ nos gatos (Janeczek et al., 2018; Llera \& Volmer, 2006; Luiz \& Heseltine, 2008).

Considerando a capacidade de recirculação enterohepática e buscando reduzir a meia vida do THC no organismo, recomenda-se a administração oral de carvão ativado, na dose de 1 a $2 \mathrm{mg} / \mathrm{kg}$, com intervalos de 8 horas, durante 24 horas (Janczyk et al., 2004; Llera \& Volmer, 2006; Wismer, 2016). Adicionalmente, Janczyk et al. (2004) recomenda a adição do catártico sorbitol na primeira dose do carvão ativado para uma melhor eliminação. Com a mesma finalidade, Llera \& Volmer (2006) sugerem a adição de sulfato de sódio ou de magnésio a $0,25 \mathrm{mg} / \mathrm{kg}$. O estado de consciência do paciente deve ser considerado, já que múltiplas doses de carvão ativado em um paciente sedado implica em um alto risco de aspiração (Parshley \& Mensching, 2014).

Por isso, paciente apresentando sintomatologia mais grave deve ser submetido a emulsão lipídica, utilizada em alimentação parenteral, podendo ser indicada para intoxicações por substâncias lipofílicas. A aplicação parenteral cria uma fase lipídica intravascular que estabelece uma movimentação da substância lipofílica dos tecidos alvo para a emulsão lipídica, reduzindo a absorção. A recomendação para a emulsão lipídica é a administração inicial de $1,5 \mathrm{~mL} / \mathrm{kg}$ em bolus lento, por 20 a 30 minutos, seguido pela administração contínua de $0,25 \mathrm{mg} / \mathrm{kg} / \mathrm{min}$ por até 60 minutos, podendo repetir esse procedimento a cada quatro horas até alcançar resposta adequada do paciente (Wismer, 2016).

Sintomas relacionados a excitação do SNC, como agitação, hiperestesia, tremores podem ser tratados com administração de acepromazina ou benzodiazepínicos, desde que não ocorra uma hipotensão concomitantemente (Wismer, 2016). A depressão do SNC ou do respiratório pode ser revertida com administração intravenosa de naloxona a $0,04 \mathrm{mg} / \mathrm{kg}$, substância que não antagoniza a apomorfina (Llera \& Volmer, 2006).

Pacientes que não desenvolverem sintomas após procedimentos para redução da absorção do THC devem permanecer em observação, sendo periodicamente monitorados quanto aos parâmetros cardíacos, respiratórios e termorregulatórios (Llera \& Volmer, 2006). Os pacientes que apresentaram sintomas requerem um cuidado maior, sendo necessário monitoramento de, além dos citados, pressão sanguínea e SNC (Parshley \& Mensching, 2014). Pacientes com afecções secundárias concomitantes possuem maiores risco de sinais mais severos (Wismer, 2016). Outro grupo de risco é formado pelos pacientes 
expostos a doses elevadas de THC que estão sujeitos a sintomas mais severos, já que se trata de uma droga dose dependente. Em casos assim, recomenda-se medidas de desintoxicação e tratamento mais enérgicos (Meola et al., 2012).

Outros cuidados podem ser necessários conforme o quadro do paciente, como a administração de fluidos intravenosos, uma opção para corrigir a hipotensão ou a desidratação (Wismer, 2016). Adicionalmente, uma fluidoterapia aquecida ou resfriada, conforme quadro apresentado pelo paciente, pode auxiliar na termorregulação (Parshley \& Mensching, 2014).

A atropina em dose $0,02 \mathrm{mg} / \mathrm{kg}$ por via intravenosa é recomendada se o paciente apresentar bradicardia. Já a oxigenioterapia auxiliará a pacientes com dificuldade ventilatórias. Pacientes com sinais que indiquem uma depressão no SNC e quadros de êmese devem receber antieméticos, para evitar uma pneumonia aspirativa (Parshley \& Mensching, 2014).

Quanto ao ambiente do animal intoxicado, a restrição de espaço pode ser benéfica para pacientes com dificuldades locomotoras, a fim de evitar traumas. É importante minimizar estímulos externos (sons, movimentos bruscos e excesso de iluminação) em hiperestésicos. Em pacientes com depressão severa ou pacientes comatosos a alternância de decúbito deve ser realizada para evitar úlceras de pressão (Parshley \& Mensching, 2014).

A ingestão de THC produz uma variedade de sinais clínicos, sendo os sinais do SNC mais predominantes, entretanto, a implantação de terapia de suporte, em um paciente sem complicações secundárias, é adequada para um prognóstico favorável (Janczyk et al., 2004; Miranda et al., 2017).

\section{Discussão}

Os autores apresentam como experiência o caso de um canino macho, 3 anos, raça Teckel, $12 \mathrm{~kg}$, atendido com o histórico de relutância para se movimentar e pouco responsivo aos estímulos externos iniciados naquela manhã, sendo que na noite anterior o paciente apresentava-se normal. $\mathrm{O}$ animal dormia dentro de casa e não referia suspeita de trauma ou contato com produto tóxico. No caminho à clínica apresentou um episódio emético mucoso no carro. No exame físico, o animal mantinha-se preferencialmente em estação, sentado com o olhar fixo, recusando-se a caminhar e respondendo somente a estímulos sonoros intensos com o movimento de cabeça. Não apresentava alteração no exame físico. Durante a investigação clínica foi implantado como tratamento a fluidoterapia com solução de cloreto de sódio 0,9\%, IV. Como a tutora tinha um compromisso, os filhos adolescentes ficaram acompanhando o paciente e nesse momento, após a ida da responsável, foi comunicado a equipe médica que havia desaparecido da casa um cigarro de Cannabis e que o paciente poderia ter acesso ao produto. A informação foi extremamente pertinente porque diante da inespecificidade dos sinais e exames laboratoriais (hemograma, função renal e hepática), o diagnóstico conclusivo não seria realizado e o caso exigiria a realizações de mais exames que seriam inconclusivos.

\section{Considerações finais}

A intoxicação por Cannabis sativa L. é uma realidade também na medicina veterinária, produzindo diversos sinais clínicos que, mesmo se concentrando em maior parte no SNC, podem se manifestar em diversos sistemas e de maneira variável, causando estímulos excitatórios ou inibitórios. A rápida implantação de uma terapia de suporte adequada, principalmente em pacientes com outras complicações concomitantes, é fundamental para proporcionar um prognóstico favorável ao paciente, por isso, é necessário que o médico veterinário saiba reconhecer e como conduzir para realizar o diagnóstico clínico, já que o método de diagnóstico rápido disponível no mercado foi desenvolvido para humanos e pode ser inválido em algumas espécies de animais de companhia, que possuem outras vias de metabolismo da droga.

\section{Referências bibliográficas}

Aguiar, A. F. L. (2018). Desenvolvimento e validação de método analítico para quantificação de canabinoides em extratos de Cannabis. Monografia - Bacharelado em Ciências Biológicas: Modalidade Médica. UFRJ, Rio de Janeiro, 
Bates, N. (2018). Exposure to drugs of abuse. Companion Animal, 23(2), 73-80. https://doi.org/10.12968/coan.2018.23.2.73

Bischoff, K. (2018). Toxicity of Drugs of Abuse. In Veterinary Toxicology (pp. 385-408). Elsevier. https://doi.org/10.1016/B978-0-12-811410-0.00022-2

Borille, B. T. (2016). Caracterização química da planta Cannabis sativa L. a partir de sementes apreendidas pela Polícia Federal no Estado do Rio Grande do Sul. Tese (Doutorado em Ciências Farmacêuticas) - Faculdade de Farmácia, Universidade Federal do Rio Grande do Sul. Porto Alegre

Botha, C. J., \& Penrith, M.-L. (2009). Potential plant poisonings in dogs and cats in southern Africa. Journal of the South African Veterinary Association, 80(2), 63-74.

Donaldson, C. W. (2002). Marijuana exposure in animals. Vet Med, 97(6), 437-439.

Farag, S., \& Kayser, O. (2017). The Cannabis Plant: Botanical Aspects. In Handbook of Cannabis and Related Pathologies (pp. 3-12). Elsevier. https://doi.org/10.1016/B978-0-12-800756-3.00001-6

Fernández, P. E., \& Pretti, R. (2017). Intoxicación por marihuana (Cannabis sativa) en perros y gatos. Revista Del Colegio Veterinario de La Provincia de Buenos Aires, 22(71), 2017.

Ferreira, M., Faraco, S., Sessegolo, G. M., \& Stefanello, C. R. (2013). Intoxicação de um canino por Cannabis sativa. Revista de Ciências Agroveterinárias, 12(Esp.), 25-26.

Fitzgerald, K. T., Bronstein, A. C., \& Newquist, K. L. (2013). Marijuana Poisoning. Topics in Companion Animal Medicine, 28(1), 8-12. https://doi.org/10.1053/j.tcam.2013.03.004

Grotenhermen, F. (2004). Pharmacology of cannabinoids. Neuroendocrinology Letters, 25(1/2), 14-23.

Hansen, D. T. K. (2006). Prevalência de intoxicações de cães e gatos em Curitiba. Dissertação (Mestrado em Ciências Veterinárias) - Universidade Federal do Paraná, Setor de Ciências Agrárias, Curitiba

Huestis, M. A. (2005). Pharmacokinetics and Metabolism of the Plant Cannabinoids, $\Delta 9$ Tetrahydrocannibinol, Cannabidiol and Cannabinol. In P. RG (Ed.), Cannabinoids (pp. 657-690). Springer-Verlag. https://doi.org/10.1007/3-540-26573-2_23

Janczyk, P., Donaldson, C. W., \& Gwaltney, S. (2004). Two hundred and thirteen cases of marijuana toxicoses in dogs. Veterinary and Human Toxicology, 46(1), 19-20.

Janeczek, A., Zawadzki, M., Szpot, P., \& Niedzwiedz, A. (2018). Marijuana intoxication in a cat. Acta Veterinaria Scandinavica, 60(1), 44. https://doi.org/10.1186/s13028-018-0398-0

Landa, L., Sulcova, A., \& Gbelec, P. (2016). The use of cannabinoids in animals and therapeutic implications for veterinary medicine: a review. Veterinární Medicína, 61(No. 3), 111-122. https://doi.org/10.17221/8762-VETMED

Lee, J. A. (2014). Pot is not for pets! Pot Is Not for Pets! https://vetgirlontherun.com/wpcontent/uploads/2014/08/Pot-is-not-for-pets-AVMA-2014.pdf

Llera, R. M., \& Volmer, P. A. (2006). Toxicologic hazards for police dogs involved in drug detection. Journal of the American Veterinary Medical Association, 228(7), 1028-1032. https://doi.org/10.2460/javma.228.7.1028

Luiz, J. A., \& Heseltine, J. (2008). Five common toxins ingested by dogs and cats. Compend Contin Educ Vet, 30(11), 578-587.

Mahaney, P. (2011). Treating Marijuana Toxicity. Veterinary Practice News. https://www.veterinarypracticenews.com/treating-marijuana-toxicity/

McGilveray, I. J. (2005). Pharmacokinetics of Cannabinoids. Pain Research and Management, 10(suppl a), 15A-22A. https://doi.org/10.1155/2005/242516

Mckinney, J. (2017) A Cannabis Breakdown: and How It Fits Into Veterinary Medicine. VetWrap, v. 11, n. 1, p. 4-9.

Meola, S. D., Tearney, C. C., Haas, S. A., Hackett, T. B., \& Mazzaferro, E. M. (2012). Evaluation of trends in marijuana toxicosis in dogs living in a state with legalized medical marijuana: 125 dogs (2005-2010). Journal of Veterinary Emergency and Critical Care, 22(6), 690-696. https://doi.org/10.1111/j.1476-4431.2012.00818.x

Miranda, A. L. S., Benito, S., \& Melo, M. M. (2017). Intoxicações de cães por drogas recreativas: maconha e cocaína. Revista Científica de Medicina Veterinária, 1-8. 
Nascimento, I. R. (2014). Identificação Química em nível molecular de amostras de maconha por ESIFT-ICR MS. . Dissertação (Mestrado em Química Orgânica) - Universidade Federal do Espírito Santo, Centro de Ciências Exatas. Vitória

Oliveira, C. D. R. (2005). Determinação de canabinóides em cabelo por microextração em fase sólida por Headspace e análise por espectrometria de massa associada à cromatografia em fase gasosa [Universidade de São Paulo]. https://doi.org/10.11606/D.9.2005.tde-21082007-160740

Parshley, L., \& Mensching, D. (2014). A Veterinary Guide to Marijuana. Washington Veterinarian, 1418.

Piedra, C. N., Arenas, G. M., García, I. M., Garduño, B. F., \& León, I. D. L.-P. (2009). La marihuana y el sistema endocanabinoide: De sus efectos recreativos a la terapéutica. Revista Biomédica, 20(2), $128-153$.

Ribeiro, J. A. C. (2014). A cannabis e suas aplicações terapêuticas. Dissertação (Mestrado em Ciências Farmacêuticas) - Universidade Fernando Pessoa, Faculdade de Ciências da Saúde. Porto

Teitler, J. B. (2009). Evaluation of a Human On-site Urine Multidrug Test for Emergency Use With Dogs. Journal of the American Animal Hospital Association, 45(2), 59-66. https://doi.org/10.5326/0450059

Turner, C. E., Elsohly, M. A., \& Boeren, E. G. (1980). Constituents of Cannabis sativa L. XVII. A review of the natural constituents. Journal of Natural Products, 43(2), 169-234.

Wismer, T. (2016) Why is My Dog Wobbly. Veterinary Team Brief. 5 (4), 31-34

Recebido: 6, de abril, 2020.

Aprovado: 19 de maio junho, 2020.

Disponível online: 4 de setembro, 2020.

Licenciamento: Este artigo é publicado na modalidade Acesso Aberto sob a licença Creative Commons Atribuição 4.0 (CC-BY 4.0), a qual permite uso irrestrito, distribuição, reprodução em qualquer meio, desde que o autor e a fonte sejam devidamente creditados. 